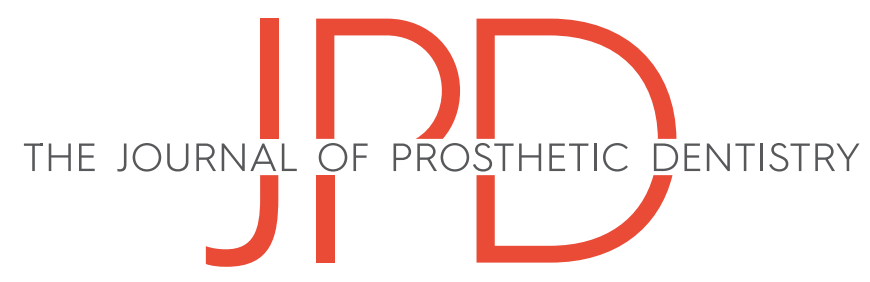

CLINICAL RESEARCH

\title{
A randomized controlled clinical trial comparing guided with nonguided implant placement: A 3-year follow-up of implant-centered outcomes
}

\author{
Lauren Bernard, DDS, ${ }^{a}$ Marjolein Vercruyssen, DDS, PhD, ${ }^{b}$ Joke Duyck, DDS, PhD, ${ }^{c}$ Reinhilde Jacobs, DDS, PhD, ${ }^{d}$ \\ Wim Teughels, DDS, PhD, ${ }^{e}$ and Marc Quirynen, DDS, $\mathrm{PhD}^{f}$
}

Osseointegrated dental implants are a treatment option for edentulous patients who seek greater stability and retention for their prosthesis. ${ }^{1,2}$ Longterm implant success data have been recorded. ${ }^{3,4}$ Guided implant placement, flapless or bone supported, was introduced more than 10 years ago, 5 and this procedure could reduce treatment time. ${ }^{6}$ Recently, a systematic review and metaanalysis by Moraschini et al, including 13 studies, reported a cumulative survival rate of $97.2 \%$. All included studies were carried out with a mucosasupported guide and immediate loading of the implants. The cumulative survival rate and marginal bone loss of the review are comparable with those of nonguided implant surgery; however, the authors reported

\begin{abstract}
Statement of problem. Implant-based prosthetic solutions can be time consuming. If implants can be placed successfully with a guide, surgery time can be reduced.

Purpose. The purpose of this randomized controlled clinical trial was to assess implant outcomes, both clinical and radiological, comparing guided with nonguided implant placement after 3 years of follow-up.

Material and methods. A total of 314 implants were placed in 72 jaws (60 participants). The jaws were randomly assigned to 1 of the 6 treatment groups: Materialise Universal/mucosa (Mat Mu), Materialise Universal/bone (Mat Bo), Facilitate/mucosa (Fac Mu), Facilitate/bone (Fac Bo), freehand navigation (Freehand), and a pilot-drill template (Templ). Radiographic and clinical parameters (bone loss, pocket probing depth, bleeding on probing, and plaque scores) were recorded at the time of implant placement, prosthesis installment (baseline), and 1-year, 2-year, and 3-year follow-up. Analysis was performed using a linear mixed model, and correction for simultaneous hypothesis was made according to Sidak $(\alpha=.05)$.
\end{abstract}

Results. Three participants left the study before the 3-year follow-up; hence, 302 implants in 69 jaws were included in this study. None of the implants failed. The mean marginal bone loss after the third year of loading was $0.7 \pm 1.3 \mathrm{~mm}$ for the guided surgery group and $0.5 \pm 0.6 \mathrm{~mm}$ for the control group. No significant intergroup or follow-up period differences were observed $(P>.05)$. In the guided surgery groups, the mean number of surfaces with bleeding on probing and plaque at 3-year follow-up was $1.7 \pm 1.5$ and $1.7 \pm 1.7$, respectively; for the control groups, this was $1.6 \pm 1.4$ and $1.6 \pm 1.6$, respectively. The mean pocket probing depth was $3.0 \pm 1.3 \mathrm{~mm}$ for the guided group and $2.6 \pm 1.0 \mathrm{~mm}$ for the control group. No significant differences were found $(P>1)$.

Conclusions. Within the limitation of this study, no statistically significant differences could be found between the guided group and the control group at the 3-year follow-up. (J Prosthet Dent

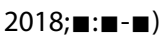

\footnotetext{
Dentsply Implants provided oral implants, prosthetic materials, and stereolithographic guides. M.Q. is Chair for Implant Supported Oral Rehabilitation at Dentsply Implants. ${ }^{a}$ Assistant, Department of Oral Health Sciences, KU Leuven \& Dentistry (Periodontology), University Hospitals Leuven, Leuven, Belgium.

bPostdoctoral Researcher, Department of Oral Health Sciences, KU Leuven \& Dentistry (Periodontology), University Hospitals Leuven, Leuven, Belgium. 'Professor and Chief, Department of Oral Health Sciences, KU Leuven \& Dentistry (Prosthetic Dentistry), University Hospitals Leuven, Leuven, Belgium. ${ }^{\mathrm{d} P r o f e s s o r}$ and Chief, OIC, OMFS IMPATH Research Group, Department of Imaging \& Pathology, KU Leuven and Oral and Maxillofacial Surgery, University Hospitals Leuven, Leuven, Belgium.

eProfessor, Department of Oral Health Sciences, KU Leuven \& Dentistry (Periodontology), University Hospitals Leuven, Leuven, Belgium.

fProfessor and Chief, Department of Oral Health Sciences, KU Leuven \& Dentistry (Periodontology), University Hospitals Leuven, Leuven, Belgium.
} 


\section{Clinical Implications}

If there is no difference in implant survival, bone loss, or clinical parameters, guided surgery can be used more commonly. This could reduce treatment time for the patient.

several surgical and prosthetic complications and a learning curve. Similar bone loss results can be found in other guided implant placement studies. ${ }^{8-10}$ These data are within the mean marginal bone loss range defined by Albrektsson et $\mathrm{al}^{11}$ as success. Pozzi et $\mathrm{al}^{12}$ compared guided versus freehand surgery with a follow-up period of 1 year. No significant difference of peri-implant bone loss could be detected between the groups.

Hultin et $\mathrm{al}^{13}$ analyzed several clinical prospective studies on clinical performance of guided implant placement in their systematic review, which concluded that for guided implant placement comparable survival rates could be expected to nonguided implant treatment. Clinical trials with at least 2 years of follow-up reach implant survival rates up to $100 \% \cdot{ }^{14-16}$ A recent systematic review reported survival rates of $97.8 \%$ or higher in the maxilla with guided placement after 1 year ${ }^{17}$ These survival rates are comparable with those of nonguided placement. ${ }^{18}$

In the present randomized controlled clinical trial, 2 guided surgery systems were used. The first system Materialise Universal (Materialise) can be used to place oral implants of different manufacturers but is carried out without depth control, and the implants are placed without guidance. The second system Facilitate (Dentsply Sirona) was designed specially to place Astra Tech implants (Dentsply Sirona) and drilling and implant placement is performed with depth control (physical stops). Data on accuracy of guided placement of the present randomized clinical trial have been published by Vercruyssen et al. ${ }^{19}$ The accuracy of the Materialise Universal system (mucosa or bone supported) and of the Facilitate system (mucosa or bone supported) was determined and compared both with freehand navigation and the use of a pilot-drill template in a delayed loading protocol. The inaccuracy of guided surgery was reported to be less than for nonguided surgery..$^{19}$ Radiographic and clinical implant parameters and patient-centered outcomes at 1-year follow-up of this study showed no differences. ${ }^{20}$

The purpose of this clinical trial was to monitor prospectively radiographic and clinical implant-centered outcomes, comparing guided versus nonguided implant placement, at a 3-year follow-up. The null hypothesis was that no difference would be found in bone loss or clinical parameters among the groups.
Table 1. Inclusion and exclusion criteria

\begin{tabular}{l}
\hline Inclusion Criteria \\
\hline 1. Provision of informed consent \\
\hline 2. Age of at least 18 years \\
\hline 3. Extraction socket healing for at least 6 months \\
\hline Exclusion criteria \\
\hline 1. Unlikely to be able to comply with study procedures \\
\hline 2. History of intravenous bisphosphonate treatment \\
\hline 3. Medical history that makes implant insertion unfavorable \\
\hline 4. Current pregnancy \\
\hline 5. Present alcohol and/or drug abuse \\
\hline 6. Major systemic diseases \\
\hline 7. Untreated, uncontrolled caries, and/or periodontal disease \\
\hline 8. History of local irradiation \\
\hline 9. Need for bone grafting and/or sinus lift in planned implant area
\end{tabular}

Table 2. Prosthesis information

\begin{tabular}{lcc}
\hline & Guided Group & Control Group \\
\hline Overdentures (maxilla/mandible) & $28(21 / 7)$ & $14(10 / 4)$ \\
\hline Fixed dental prosthesis (maxilla/mandible) & $20(8 / 12)$ & $10(1 / 9)$ \\
\hline
\end{tabular}

\section{MATERIAL AND METHODS}

This study was designed as a randomized controlled clinical trial. Candidates for the study were selected from patients in need of complete rehabilitation of the mandible or maxilla at the units of restorative dentistry or periodontology at the University Hospital Leuven. The information about the study participants and groups is shown in Tables 1 and 2. All participants were assigned to 1 of the 6 groups, 4 guided groups (MatMu/MatBo/ $\mathrm{FacMu} / \mathrm{FacBo}$ ), the experimental group, and 2 nonguided groups (Freehand/Temp), the control group. Randomization was performed by a randomization table provided by an external statistician. Determination of the sample size was based on the accuracy data. ${ }^{19}$

The study was approved by the ethical committee of the University Hospital (B32220095376) and by the Federal Agency for Medicines and Health Products (Health Authority). This study was registered with the U.S. National Institutes of Health Clinical Trials Registry (NCT03041584). All participants signed a consent form.

All participants underwent multislice computer tomography (Somatom Definition Flash, at $120 \mathrm{kV}$ and 90 mAs, 0.6-mm-slice thickness, voxel size 330 lm; Siemens Healthcare $\mathrm{GmbH}$ ) with a scan prosthesis and occlusal index positioned in the mouth. The implants were planned in 3D software (Simplant; Materialise Dental) by one investigator (M.V.), a member of the periodontology department, in the optimal position considering both the alveolar process and the prosthetic demands. At that moment, the jaws were randomized with the randomization table to 1 of the 6 groups. 

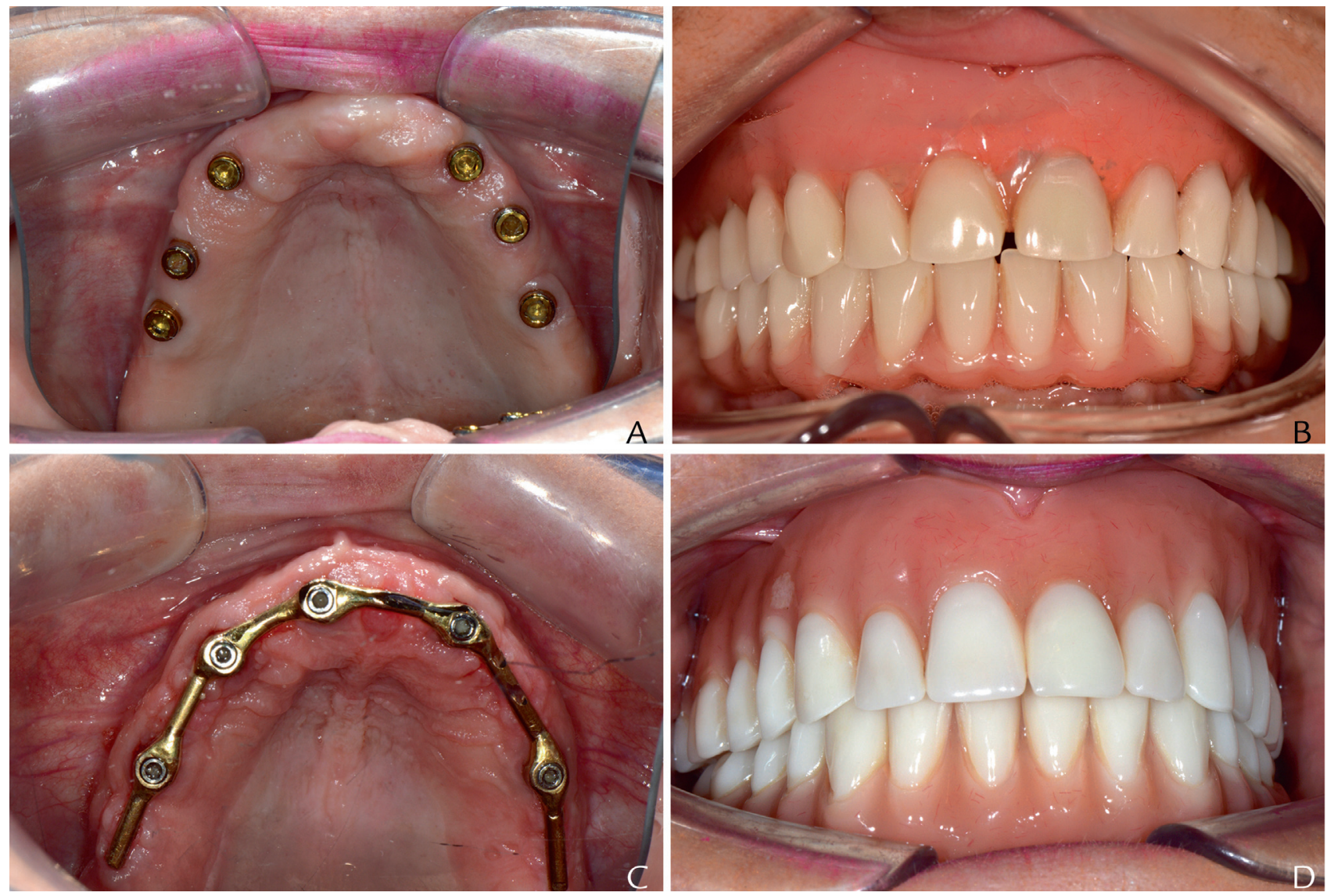

Figure 1. Clinical photographs of prosthesis and abutments. A. Non-splinted attachment system for an overdenture (Locator). B. Removable prostheses in the maxilla, fixed prostheses in the mandible. C. Splinted attachment system for an overdenture (bar). D. Removable prostheses in the maxilla, overdenture in the mandible.

The surgery was performed under local anesthesia at the University Hospital. All patients received 4 to 6 implants, with diameters of 3.5 or $4 \mathrm{~mm}$ and lengths ranging from 8 to $15 \mathrm{~mm}$. Images from software planning and rough distance calculations were applied for the freehand navigation group (Freehand), a surgical stent with pilot drilling for the template group (Templ), a mucosa-supported stereolithographic guide for the Materialise Dental mucosa (Mat $\mathrm{Mu}$ ) and Facilitate mucosa group (Fac $\mathrm{Mu}$ ), and a bone-supported stereolithographic guide for the Materialise Dental bone (Mat Bo) and Facilitate bone group (Fac Bo).

The implants were immediately loaded in 1 participant from the guided group with an interim fixed dental prosthesis. This participant received the definitive prosthesis after 6 months. All other participants had healing abutments placed and received the definitive prosthesis 3 months after implant insertion. After placement of the uni-abutments (Astra Tech; Dentsply Sirona), an impression was made (Impregum; $3 \mathrm{M}$ ESPE). The metal framework was fabricated in the laboratory and evaluated for fit before the denture teeth were arranged and the gingival tissues were modeled in wax. Occlusion was evaluated and adjusted if necessary.

After the tooth arrangement was approved by the patient, the prosthesis was processed (Table 2). All overdentures were supported by 4 implants, as were the fixed prostheses in the mandible. One prosthesis in the mandible was placed on 6 implants. In the maxilla, the fixed prostheses were supported by 6 implants, except for one supported by 4 implants. More detailed information about the planning and the prosthetic procedures can be found in a previous article. ${ }^{21}$

The follow-up visits were after evaluation of the definitive prosthesis framework (baseline) and 1, 2, and 3 years after delivery of the definitive prosthesis (Fig. 1). Clinical parameters were evaluated at these visits except for the plaque score, which was not measured at baseline. All clinical parameters were scored at the 4 sites (mesial/distal/ buccal/lingual) of each implant. Plaque was scored as absent (0) or present (1). Bleeding on probing (BoP) was recorded 20 seconds after gentle probing, with following scores: no bleeding (0), bleeding (1). Probing pocket depth was recorded with the implant/abutment junction as reference level, using a Merritt-B (Hu-Friedy) periodontal probe. 
Table 3. Participants and implant features

\begin{tabular}{lcccccc}
\hline & Mat Mu & Mat Bo & Fac Mu & Fac Bo & Freehand & Templ \\
\hline Total participants & 11 & 12 & 11 & 11 & 12 & 12 \\
\hline Sex (male/female) & $5 / 7$ & $4 / 8$ & $6 / 6$ & $4 / 8$ & $4 / 8$ & $8 / 4$ \\
\hline Age (range)* & $38-78$ & $31-72$ & $46-74$ & $43-65$ & $39-72$ & $40-75$ \\
\hline Smokers & 0 & 3 & 2 & 1 & 0 & 2 \\
\hline Jaw (maxillary/mandibular) & $6 / 6$ & $3 / 9$ & $5 / 7$ & $6 / 6$ & $9 / 3$ & $4 / 8$ \\
\hline Prosthesis (fixed/overdenture) & $7 / 5$ & $3 / 9$ & $2 / 10$ & $8 / 4$ & $6 / 6$ & $4 / 8$ \\
\hline No. of implants placed & 55 & 53 & 52 & 52 & 51 & 51 \\
\hline No. of implants analyzed at 3-y follow-up & 51 & 53 & 48 & 48 & 51 & 51 \\
\hline No. of implants placed, 1 stage/2 staged & $51 / 4$ & $35 / 18$ & $48 / 4$ & $47 / 5$ & $41 / 10$ & $23 / 28$ \\
\hline Implant diameter (3.5/4 mm) & $15 / 40$ & $7 / 46$ & $21 / 31$ & $31 / 21$ & $18 / 33$ & $47 / 4$ \\
\hline Implant length (8/9/11/13/15 mm) & $0 / 3 / 28 / 23 / 1$ & $1 / 7 / 17 / 17 / 11$ & $5 / 9 / 20 / 18$ & $0 / 3 / 12 / 28 / 9$ & $0 / 1 / 12 / 16 / 22$ & $2 / 10 / 17 / 18 / 4$ \\
\hline
\end{tabular}

Fac Bo, Facilitate/bone; Fac Mu, Facilitate/mucosa; Freehand, freehand navigation; Mat Bo, Materialise Universal/bone; Mat Mu, Materialise Universal/ mucosa; Templ, surgical template. *At time of implant placement.

Postoperative radiographs were made at loading (baseline) and at the 1-, 2-, and 3-year follow-up. All measurements were performed by an independent examiner (L.B.), who was not involved in the treatment process. Bone loss over time was calculated for each mesial and distal site.

The statistical analysis was similar to that performed at 1-year follow-up.9 Peri-implant bone-level changes were considered to be efficacy variables. Clinical data (pocket probing depth, BoP, and plaque scores) were considered as descriptors. The outcome variable was the peri-implant bone-level change.

Explanatory variables were the difference in marginal bone loss between the different treatment groups and the evolution of the implant-centered outcomes over time. The implant-centered variables were analyzed with a linear mixed model taking the explanatory variables as fixed factors and patient as a random factor. Residual dot plots and normal quantile plots were used to assess the assumptions of the model. Contrasts were built to test the specific hypotheses and a correction for simultaneous hypothesis testing was made according to $\operatorname{Sidak}^{22}(\alpha=.05)$. A post hoc power analysis was performed to calculate the data that would be needed to find the current difference as significant with a power of $50 \% .{ }^{23}$

\section{RESULTS}

A total of 314 implants (Astra Tech TX; Dentsply Sirona) were inserted. In every patient, 4 to 6 implants were placed. Three patients dropped out of the study. One patient of the guided Fac Bo group died. Two patients did not comply with the recall schedule, one from the guided Fac Mu group and one from the guided Mat $\mathrm{Mu}$ group. All other participants complied with the recall schedule. The implants were placed between August 2009 and June 2012. Forty-five jaws were followed up in the experimental group and 24 , in the control group. A total of 302 implants were followed up during 3 years of

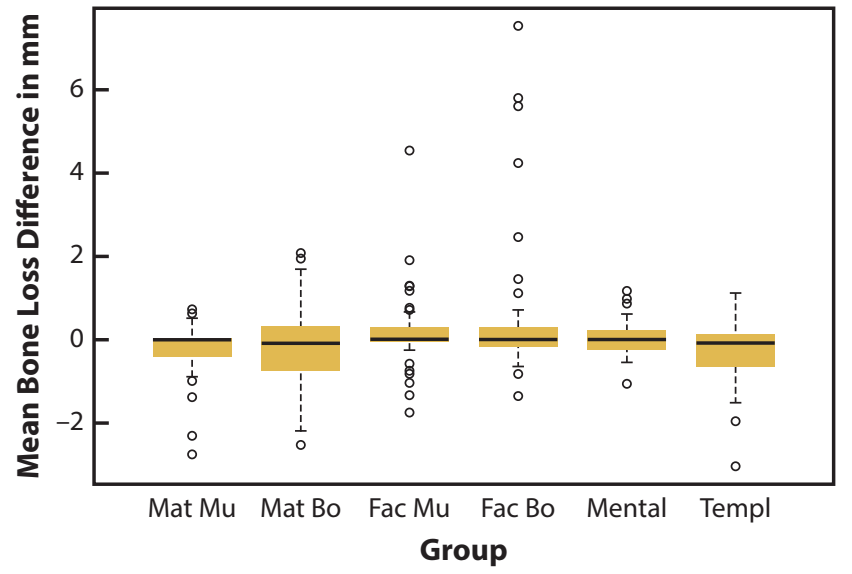

Figure 2. Box plot mean bone loss difference at 3 years compared with baseline. Fac Bo, Facilitate/bone; Fac Mu, Facilitate/mucosa; Mental, freehand navigation; Mat Bo, Materialise Universal/bone; Mat Mu, Materialise Universal/mucosa; Templ, surgical template.

loading. No implant was lost during the study period. Details on patient and implant features are shown in Table 3.

The difference in mesial and distal bone loss for all the implants between baseline and 3 years of loading is shown in Figures 2, 3. The mean bone loss values were $0.6 \pm 1.1 \mathrm{~mm}$ and $0.7 \pm 1.3 \mathrm{~mm}$ for the experimental group and $0.4 \pm 0.7 \mathrm{~mm}$ and $0.5 \pm 0.6 \mathrm{~mm}$ for the control group at 2- and 3-year follow-up. Table 4 shows the mean bone loss values of all groups over all follow-up periods. No significant intergroup differences could be observed $(P>.05)$. Compared with the baseline for the experimental group at 3-year follow-up, a bone loss difference of 0.1 $\pm 1.2 \mathrm{~mm}$ was found, and for the control group, a bone loss difference of $-0.1 \pm 0.6 \mathrm{~mm}$ was found. Neither was statistically significantly different. Smokers had significantly more bone loss than nonsmokers regardless of the group, $0.9 \pm 0.8 \mathrm{~mm}(P=.012)$.

In the experimental group, the mean number of surfaces per implant with plaque at 3-year follow-up was 


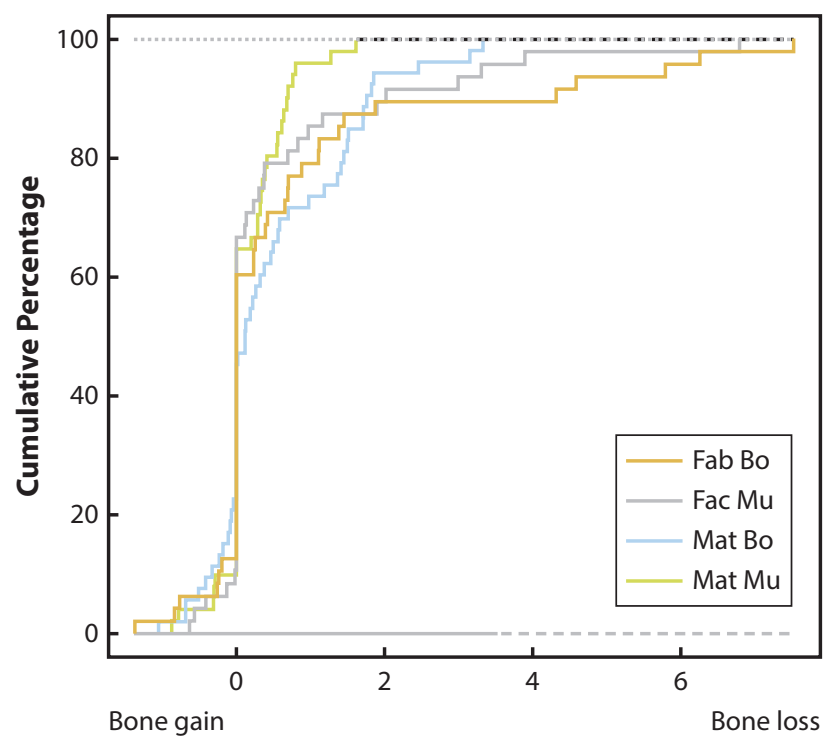

A

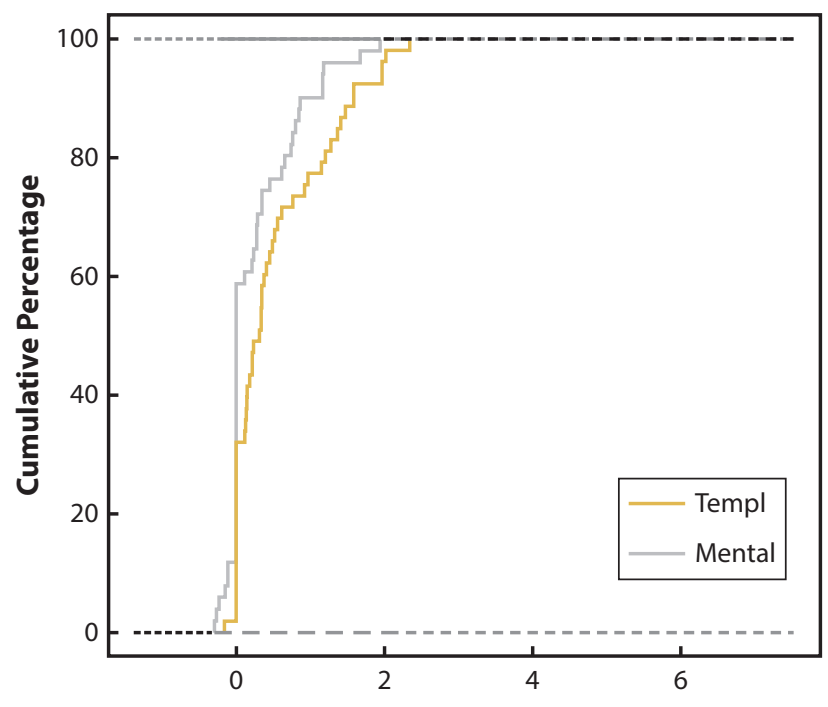

Bone gain

Bone loss

B

Figure 3. Cumulative percentage bone loss: baseline compared with 3-year follow-up. Fac Bo, Facilitate/bone; Fac Mu, Facilitate/mucosa; Mental, freehand navigation; Mat Bo, Materialise Universal/bone; Mat $\mathrm{Mu}$, Materialise Universal/mucosa; Templ, surgical template.

$1.7 \pm 1.7$ and $1.6 \pm 1.6$ for the control group, compared with $1.1 \pm 1.2$ for the experimental group and $1.8 \pm 1.6$ for the control group at 1-year follow-up. Most of the implant sites showed pocket probing depths of $\leq 4 \mathrm{~mm}$ at the 3-year follow-up. The mean probing pocket depth for the experimental groups was $3.0 \pm 1.3 \mathrm{~mm}$ at 3 -year follow-up, compared with the $2.6 \pm 0.9 \mathrm{~mm}$ at baseline. For the control groups, this was $2.6 \pm 1.0 \mathrm{~mm}$ for the 3 year follow-up and $2.3 \pm 0.8 \mathrm{~mm}$ at baseline.

At baseline, the mean number of surfaces with BoP in the control group was $1.1 \pm 1.0$ and in the experimental
Table 4. Bone loss ( $\mathrm{mm}$ ) (mean \pm standard deviation)

\begin{tabular}{lcccccc}
\hline Year & Mat Mu & Mat Bo & Fac Mu & Fac Bo & Freehand & Templ \\
\hline 1 & $0.5 \pm 0.6$ & $1.0 \pm 1.0$ & $0.6 \pm 1.0$ & $0.5 \pm 0.7$ & $0.3 \pm 0.4$ & $0.9 \pm 1.0$ \\
\hline 2 & $0.3 \pm 0.5$ & $0.7 \pm 0.8$ & $0.6 \pm 1.3$ & $0.8 \pm 1.5$ & $0.3 \pm 0.5$ & $0.5 \pm 0.9$ \\
\hline 3 & $0.2 \pm 0.4$ & $0.9 \pm 1.0$ & $0.7 \pm 1.3$ & $0.9 \pm 1.9$ & $0.3 \pm 0.5$ & $0.6 \pm 0.7$
\end{tabular}

Fac Bo, Facilitate/bone; Fac Mu, Facilitate/mucosa; Freehand, freehand navigation; Mat Bo, Materialise Universal/bone; Mat Mu, Materialise Universal/mucosa; Templ, surgical template. No significant differences in bone loss were observed (P>.05).

Table 5. Bleeding on probing, probing pocket depth, and plaque as measured at different time points (mean \pm standard deviation)

\begin{tabular}{ccc}
\hline BoP & Guided, $\mathbf{n = 4 5}$ & Nonguided, $\mathbf{n = 2 4}$ \\
\hline Baseline & & \\
\hline 1-y follow-up & $1.3 \pm 1.3$ & $1.1 \pm 1.0$ \\
\hline 2-y follow-up & $1.1 \pm 1.3$ & $1.4 \pm 1.3$ \\
\hline 3-y follow-up & $1.7 \pm 1.5$ & $1.2 \pm 1.4$ \\
\hline PPD & $1.7 \pm 1.5$ & $1.6 \pm 1.4$ \\
\hline Baseline & & \\
\hline 1-y follow-up & $2.6 \pm 0.9$ & $2.3 \pm 0.8$ \\
\hline 2-y follow-up & $2.8 \pm 1.1$ & $2.5 \pm 0.9$ \\
\hline 3-y follow-up & $3.0 \pm 1.1$ & $2.4 \pm 0.7$ \\
\hline Plaque & $3.0 \pm 1.3$ & $2.6 \pm 1.0$ \\
\hline 1-y follow-up & & $1.8 \pm 1.6$ \\
\hline 2-y Follow-up & $1.1 \pm 1.2$ & $1.8 \pm 1.7$ \\
\hline 3-y Follow-up & $1.1 \pm 1.5$ & $1.6 \pm 1.6$ \\
\hline
\end{tabular}

BoP, bleeding on probing in number of surfaces per implant (max 4 surfaces: mesial, distal, buccal, and oral); $\mathrm{n}$, total number of jaws; PPD, probing pocket depth in $\mathrm{mm}$; plaque in number of surfaces per implant.

group, $1.3 \pm 1.3$. At 3 -year follow-up, this was $1.6 \pm 1.4$ for the control group and $1.7 \pm 1.5$ for the experimental group. No statistical difference between groups or followup period was found (P>.1) (Table 5).

\section{DISCUSSION}

The null hypothesis that no difference in bone loss or clinical parameters wound be found between the groups was accepted. Three participants were lost during follow-up; however, no implants were reported to be lost. In the remaining patients, of the $302 \mathrm{im}$ plants that were followed up for 3 years, none were lost. This high survival rate is consistent with similar clinical trials, ${ }^{14-16}$ which may have been because of strict inclusion and exclusion criteria, together with comprehensive diagnosis and screening. Three studies had a follow-up period of at least 2 years ${ }^{14-16}$ One study that reported on survival rate of guided implantation had a follow-up period up to 5 years ${ }^{24}$ All other included studies had observational periods of less than 2 years. The reported implant survival rate after 1 year ranged between $89 \%$ and $100 \%$ (study mean 97\%), which is comparable with the systematic review of Moraschini et $\mathrm{al}^{7}$ describing a cumulative survival rate of $97.2 \%$. In recent systematic review of 
guided implant placement in the maxilla, all studies reported survival rates of $97.8 \%$ or higher after 1 year ${ }^{17}$ Implants placed with a guide appear to have a survival rate comparable with those placed without a guide with 1-year survival rates of $94 \% .^{18}$

The present study could not detect a difference in mean bone loss nor in clinical parameters between the individual treatment groups or between guided or nonguided implant placement. A post hoc power analysis measured an $\mathrm{N}$-factor of $15.14 .^{23}$ The $\mathrm{N}$-factor is the percentage of extra data points needed to achieve a clinically significant difference, considering that when the data set is expanded, the variability in data would remain the same.

The results for mean marginal bone loss of the guided groups, $0.7 \pm 1.3 \mathrm{~mm}$, are comparable with those of the systematic review and meta-analysis carried out by Moraschini et $\mathrm{al}_{1}^{7}$ who reported a mean marginal bone loss of $1.5 \pm 0.4 \mathrm{~mm}$ in a follow-up period between 1 and 4 years. Other studies on guided implant placement with a follow-up of less than 3 years reported mean bone loss of $0.5 \pm 1.0 \mathrm{~mm},{ }^{8} 1.2 \pm 1.6 \mathrm{~mm},{ }^{9}$ and 1.4 $\pm 1.6 \mathrm{~mm} .{ }^{10}$

The present trial is consistent with the results by Pozzi et al. ${ }^{12}$ Both trails could not detect a significant difference of peri-implant bone loss between guided and nonguided groups at 1-year follow-up or up to 3 -year followup in the present study.

There were only 7 smokers in this trial, but a significant effect of smoking could be observed. For all treatment groups, smokers had more bone loss $(P=.012)$ than nonsmokers. These data are consistent with those of Sanna et $\mathrm{al}_{1}{ }^{24}$ who reported $0.8 \pm 1.1 \mathrm{~mm}$ in nonsmokers and $1.1 \pm 1.4 \mathrm{~mm}$ in smokers at 1 year, similar to the data reported by D'haese et $\mathrm{al}_{,}^{8} 0.3 \pm 0.3 \mathrm{~mm}$, smokers, and $1.0 \pm 1.4 \mathrm{~mm}$, nonsmokers.

In this randomized controlled clinical study, no failures were reported. A possible cause for implant failures mentioned in the systematic review by Hultin et $\mathrm{al}^{13}$ is bruxism. ${ }^{25}$ In this study, no influence of bruxism could be found on implant survival nor on marginal bone level.

The present study had insufficient power to measure survival differences between the groups. A longer followup time and larger groups are suggested for future research.

\section{CONCLUSIONS}

Within the limitations of this clinical study, the following conclusions were drawn:

1. No statistically differences could be found between the guided group and the control group at 3-year follow-up.
2. A significant difference in bone loss was found in smokers, regardless of the treatment group.

\section{REFERENCES}

1. Besimo C, Graber G. A new concept of overdentures with telescope crowns on osseointegrated implants. Int J Periodontics Restorative Dent 1994;14: 486-95.

2. Romanos GE. Surgical and prosthetic concepts for predictable immediate loading of oral implants. J Calif Dent Assoc 2004;32:991-1001.

3. Jung RE, Zembic A, Pjetursson BE, Zwahlen M, Thoma DS. Systematic review of the survival rate and the incidence of biological, technical, and aesthetic complications of single crowns on implants reported in longitudinal studies with a mean follow-up of 5 years. Clin Oral Implants Res 2012;23: 2-21.

4. Pjetursson BE, Thoma D, Jung R, Zwahlen M, Zembic A. A systematic review of the survival and complication rates of implant- supported fixed dental prostheses (FDPs) after a mean observation period of at least 5 years. Clin Oral Implants Res 2012;23:22-38.

5. Vercruyssen M, Laleman I, Jacobs R, Quirynen M. Computer-supported implant planning and guided surgery: a narrative review. Clin Oral Implants Res 2015;26:69-76.

6. Colombo M, Mangano C, Mijiritsky E, Krebs M, Hauschild U, Fortin T. Clinical applications and effectiveness of guided implant surgery: a critical review based on randomized controlled trials. BMC Oral Health 2017; 17:150.

7. Moraschini V, Velloso G, Luz D, Barboza EP. Implant survival rates, marginal bone level changes, and complications in full-mouth rehabilitation with

flapless computer-guided surgery: a systematic review and meta-analysis. Int J Oral Maxillofac Surg 2015;44:892-901.

8. D'haese I, Vervaeke S, Verbanck N, De Bruyn H. Clinical and radiographic outcome of implants placed using stereolithographic guided surgery: a prospective monocenter study. Int J Oral Maxillofac Implants 2013;28:205-15.

9. Marra R, Acocella A, Rispoli A, Sacco R, Ganz SD, Blasi A. Full-mouth rehabilitation with immediate loading of implants inserted with computerguided flap-less surgery: a 3-year multicenter clinical evaluation with oral health impact profile. Implant Dent 2013;22:444-52.

10. Komivama A, Hultin M, Nasstrom K, Benchimol D, Klinge B. Soft tissue conditions and marginal bone changes around immediately loaded implants inserted in edentate jaws following computer guided treatment planning and flapless surgery: a $>1$-year clinical follow-up study. Clin Implant Dent Relat Res 2012;14:157-69.

11. Albrektsson T, Zarb G, Worthington P, Eriksson AR. The long-term efficacy of currently used dental implants: a review and proposed criteria of success. Int J Oral Maxillofac Implants 1986;1:11-25.

12. Pozzi A, Tallarico M, Marchetti M, Scarfo B, Esposito M. Computer-guided versus free-hand placement of immediately loaded dental implants: 1-year post-loading results of a multicentre randomised controlled trial. Eur J Oral Implantol 2014;7:229-42.

13. Hultin M, Svensson KG, Trulsson M. Clinical advantages of computerguided implant placement: a systematic review. Clin Oral Implants Res 2012;23:124-35.

14. Nkenke E, Eitner S, Radespiel-Troger M, Vairaktaris E, Neukam FW, Fenner M. Patient-centred outcomes comparing transmucosal implant placement with an open approach in the maxilla: a prospective, non-randomized pilot study. Clin Oral Implants Res 2007:18:197-203

15. Danza M, Zollino I, Carinci F. Comparison between implants inserted with and without computer planning and custom model coordination. J Craniofac Surg 2009;20:1086-92

16. Berdougo M, Fortin T, Blanchet E, Isidori M, Bosson J-L. Flapless implant surgery using an image-guided system. A 1- to 4-year retrospective multicenter comparative clinical study. Clin Implant Dent Relat Res 2010;12: 142-52.

17. Laleman I, Bernard L, Vercruyssen M, Jacobs R, Bornstein MM, Quirynen M. Guided implant surgery in the edentulous maxilla: a systematic review. Int J Oral Maxillofac Implants 2016;31:103-17.

18. Lambert FE, Weber H-P, Susarla SM, Belser UC, Gallucci GO. Descriptive analysis of implant and prosthodontic survival rates with fixed implant supported rehabilitations in the edentulous maxilla. J Periodontol 2009;80: 1220-30.

19. Vercruyssen M, Cox C, Coucke W, Naert I, Jacobs R, Quirynen M. A randomized clinical trial comparing guided implant surgery (bone- or mucosa- supported) with mental navigation or the use of a pilot-drill template. J Clin Periodontol 2014;41:717-23.

20. Vercruyssen M, Van De Wiele G, Teughels W, Naert I, Jacobs R, Quirynen M. Implant- and patient-centred outcomes of guided surgery, a 1-year follow-up: an RCT comparing guided surgery with conventional implant placement. J Clin Periodontol 2014;41:1154-60. 
21. Vercruyssen M, Coucke W, Naert I, Jacobs R, Teughels W, Quirynen M. Depth and lateral deviations in guided implant surgery: an RCT comparing guided surgery with mental navigation or the use of a pilot-drill template. Clin Oral Implants Res 2015;26:1315-20.

22. Sidak Z. Rectangular confidence regions for the means of multivariate normal distributions. I Am Stat Assoc 1967;62:626-33.

23. Kaplan D. A rejoinder on evaluating and modifying covariance structure models. Multivariate Behav Res 1990;25:197-204.

24. Sanna AM, Molly L, van Steenberghe D. Immediately loaded CAD-CAM manufactured fixed complete dentures using flapless implant placement procedures: a cohort study of consecutive patients. J Prosthet Dent 2007;97: 331-9.

25. Malo P, de Araujo Nobre M, Lopes A. The use of computer-guided flapless implant surgery and four implants placed in immediate function to support fixed denture: preliminary results after a mean follow-up period of thirteen months. J Prosthet Dent 2007;97:S26-34.

\section{Corresponding author:}

Lauren Bernard

Katholieke Universiteit Leuven

UZ St. Raphael, Department of Periodontology

Kapucijnenvoer 33, B-3000 Leuven

BELGIUM

Email: info@parobernard.be

Copyright () 2018 by the Editorial Council for The Journal of Prosthetic Dentistry. https://doi.org/10.1016/j.prosdent.2018.09.004 